\title{
Solving self-absorption in fluorescence
}

\author{
R. M. Trevorah, C. T. Chantler \\ School of Physics, University of Melbourne \\ ryanmt@student.unimelb.edu.au
}

95\% of XAS research uses measurement of secondary fluorescence photons, which suffers from uncalibrated detector efficiencies and a dominant systematic of self-absorption of the fluorescence photon, which compromises accuracy, analysis, and insight. We have developed, coded and implemented a novel self-consistent method to correct for self-absorption seen in high-energy fluorescence X-ray measurements $[1,2]$. This method and the resulting software package can be applied to any fluorescence data set.

The complexes considered here, n-pr and i-pr, have been shown to have local metal environments with approximate tetrahedral and square planar coordination geometries using transmission mode XAS [3]. This provides an excellent test of fluorescent multi pixel data and demonstrates the merit of using complimentary techniques to confirm molecular geometries.

A dramatic discrepancy is seen between the spectra from the two measurements. This is due to the self-absorption systematic and also to uncalibrated detector efficiencies in the fluorescence measurement. While the detector efficiency can be corrected for, there is currently no self-consistent method for removing the effect of self-absorption from the spectra. In this work, we predict to high accuracy the magnitude of dispersion and energy functional due to self-absorption. As a result, the dispersion is greatly reduced, and the spectral shape follows the classic XAS trend (Fig. 1). The results presented here demonstrate a dramatic improvement over any previous work in the literature. Our modern theory is of the best quality, allowing our self-absorption correction to be applied to any fluorescence XAS data set and opening up an entire class of experimental investigation.

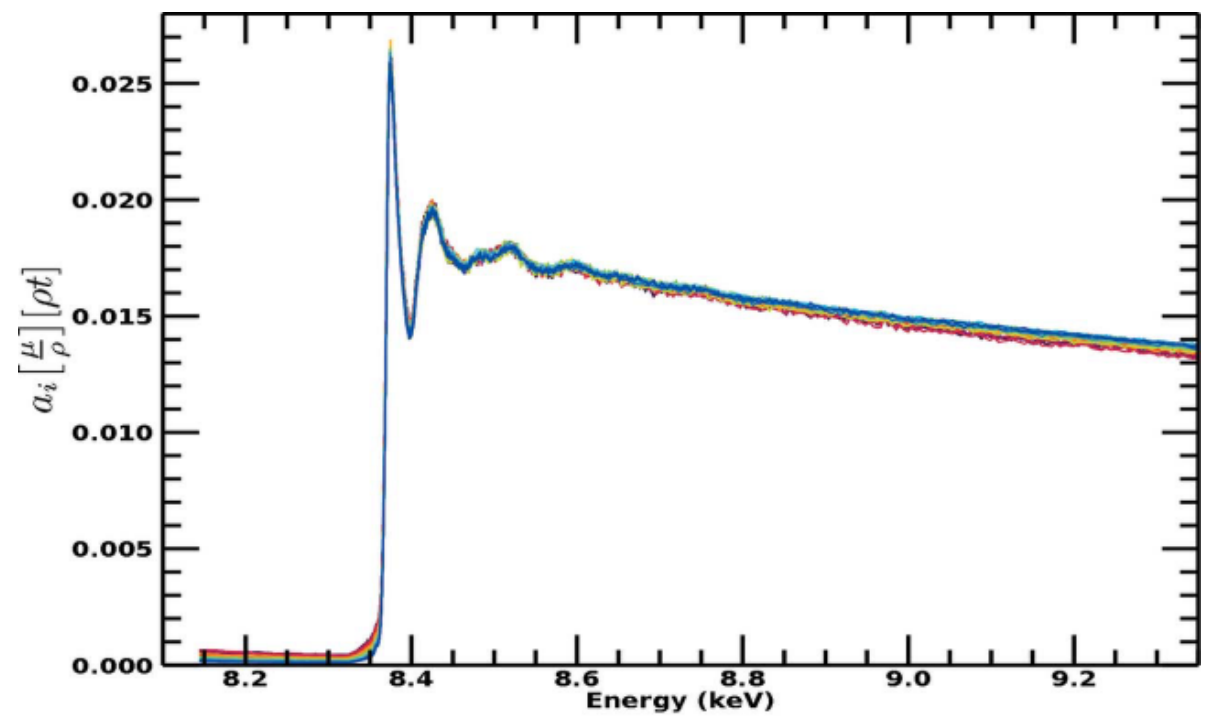

Figure 1. Ni i-pr SeAFFluX-corrected spectra with a scaled overplot of the transmission XAS spectra.

[1] R. M. Trevorah, et al., IUCr J, 6, 586 (2019)

[2] R. M. Trevorah, et al., J. Phys. Chem. A, 124, 8, 1634 (2020)

[3] C. T. Chantler, et al., J. Synchrotron Radiat. 22, 1008 (2015).

Keywords: XAFS; self-absorption; fluorescence 